\title{
Theory of electron-hydrogen-atom collisions in the presence of a laser field
}

\author{
H. S. Brandi and Belita Koiller \\ Departamento de Física, Pontifícia Universidade Católica, Cx.P. 38071, Rio de Janeiro, RJ, Brazil \\ H. G. P. Lins de Barros \\ Centro Brasileiro de Pesquisas Físicas, 20.000, Rio de Janeiro, RJ, Brazil \\ L. C. M. Miranda and J. J. Castro* \\ Instituto de Física, Universidade Estadual de Campinas, 13:000, Campinas, S.P., Brazil
}

(Received 10 August 1977)

\begin{abstract}
Expressions for the scattering amplitude of multiphoton processes occurring during the collision of electrons with a hydrogen atom in the presence of an electromagnetic field have been obtained in several approximations: the momentum translation, the space translation, and the Keldysh approximation for the bound-electron wave function. We also propose a perturbation approach based on the space-translation approximation to treat the hydrogen-atom bound states. Our results show that it is of fundamental importance to consider the modifications of bound states due to the presence of the field.
\end{abstract}

\section{INTRODUCTION}

In the last few years a number of works have been done concerning the electron scattering by atoms in the presence of an electromagnetic field (EMF). ${ }^{1-11}$

This process is very important in the study of plasma heating by electromagnetic waves, ${ }^{1-3,9,10}$ in the study of atoms excited resonantly by lasers, ${ }^{4-8,11}$ gas breakdown, etc. One of the principal difficulties involved in the theoretical treatment of these scattering processes is the description of the bound states in an EMF. In particular, if the EMF is strong enough, the excited states of the atom can be so modified that to ignore this change may yield nonreliable results for the multiphoton scattering amplitudes.

In this work we present a systematic discussion of different approximation to treat electron scattering by a hydrogen atom in the presence of an EMF, and we obtain the expressions for the multiphoton transition amplitude using the Green'sfunction formalism. We remark that this same problem has been studied previously by Perelman and Kovarskii, ${ }^{1}$ Bhakar and Choudhury, ${ }^{3}$ Macek and Hertel, ${ }^{6}$ Gersten and Mittleman, ${ }^{7}$ Mittleman, ${ }^{8}$ and Mohan. ${ }^{11}$ Our aim is to compare . different approaches proposed for the treatment of systems in an EMF, and therefore present conclusions about these approximations.

In Sec. II we present the space-translation transformation $^{9-12}$ for the Hamiltonian and give a detailed description of the general formalism yielding to the scattering amplitude: the main difficulty is to treat the bound state adequately, which is of fundamental importance in the final results. We propose a perturbation scheme for a consistent treatment of the bound states in an EMF and carry it out to first order (first-order space-translation approximation or STA1).

In Sec. III the formalism developed in the preceding section is applied to the original Hamiltonian, and two different approximations are used to describe the bound states in an EMF, namely, the momentum-translation approximation (MTA) ${ }^{13}$ and the Keldysh approximation ${ }^{14}(\mathrm{KA})$. According to Reiss, ${ }^{13}$ the MTA gives a good description of the atomic system in an EMF as long as $\omega \ll \epsilon$, where $\omega$ is the field frequency and $\epsilon$ is a typical energy of the atomic process; some criticism concerning the MTA has appeared in the literatur $\mathrm{e}^{15,16}$ and we comment on this. The KA was previously . used by Bhakar and Choudhury ${ }^{3}$ in the treatment of this same problem. In Sec. IV we assume the Born approximation and obtain simple expressions for the scattering amplitude in the different approximations considered here. We present the comparison of our results and those of other authors together with our conclusions in Sec. V.

\section{USE OF THE SPACE-TRANSLATION APPROXIMATION TO OBTAIN THE SCATTERING AMPLITUDE}

The Hamiltonian for the scattering of electron 2 from the bound state of electron 1 and proton 3 at the origin, in the presence of an EMF represented by a vector potential $\vec{A}(\hbar=c=1)$ is

$$
\begin{aligned}
H= & (1 / 2 m)\left(\overrightarrow{\mathrm{p}}_{1}-e \overrightarrow{\mathrm{A}}\right)^{2}+V_{2}\left(\overrightarrow{\mathrm{r}}_{1}\right)+(1 / 2 m)\left(\overrightarrow{\mathrm{p}}_{2}-e \overrightarrow{\mathrm{A}}\right)^{2} \\
& +V_{1}\left(\overrightarrow{\mathrm{r}}_{2}\right)+V_{3}\left(\overrightarrow{\mathrm{r}}_{1}, \overrightarrow{\mathrm{r}}_{2}\right)
\end{aligned}
$$

where 


$$
\begin{aligned}
& \overrightarrow{\mathrm{p}}_{1}=-i \vec{\nabla}_{1}, \quad \overrightarrow{\mathrm{p}}_{2}=-i \vec{\nabla}_{2}, \\
& V_{1}=-e^{2} / r_{2}, \quad V_{2}=-e^{2} / r_{1}, \quad V_{3}=e^{2} /\left|\overrightarrow{\mathrm{r}}_{1}-\overrightarrow{\mathrm{r}}_{2}\right| .
\end{aligned}
$$

The subscript $i$ in the two-body spherically symmetric potential $V_{i}$ denotes that the $i$ th particle is not interacting. We assume throughout this paper the dipole approximation for the EMF, so that $\vec{A}$ is a time-dependent spatially homogeneous vector potential:

$$
\overrightarrow{\mathrm{A}}=\overrightarrow{\mathrm{A}}_{0} \cos \omega t \text {. }
$$

We introduce the space-translation transformation $^{9-12}$

$$
T=\exp \left\{-i\left[\vec{\alpha}(t) \cdot \overrightarrow{\mathrm{p}}_{1}+\eta(t)\right]\right\},
$$

where

$\vec{\alpha}(t)=-\frac{e}{m} \int^{t} d t^{\prime} \overrightarrow{\mathrm{A}}\left(t^{\prime}\right)=-\frac{e \overrightarrow{\mathrm{A}}_{0}}{m \omega} \sin \omega t=-\vec{\alpha}_{0} \sin \omega t$,

$\eta(t)=\frac{e^{2}}{2 m} \int^{t} d t^{\prime} A^{2}\left(t^{\prime}\right)$.

Under this transformation the time-dependent Schrodinger equation takes the form

$$
\begin{aligned}
\tilde{H} \Psi\left(\overrightarrow{\mathrm{r}}_{1}, \overrightarrow{\mathrm{r}}_{2}, t\right)= & \left(\frac{p_{1}^{2}}{2 m}+V_{2}\left[\overrightarrow{\mathrm{r}}_{1}+\vec{\alpha}(t)\right]+\frac{1}{2 m}\left(\overrightarrow{\mathrm{p}}_{2}+e \overrightarrow{\mathrm{A}}\right)^{2}\right. \\
& \left.+V_{1}\left(\overrightarrow{\mathrm{r}}_{2}\right)+V_{3}\left(\overrightarrow{\mathrm{r}}_{1}+\vec{\alpha}(t), \overrightarrow{\mathrm{r}}_{2}\right)\right) \Psi\left(\overrightarrow{\mathrm{r}}_{1}, \overrightarrow{\mathrm{r}}_{2}, t\right) \\
= & i \frac{\partial \Psi\left(\overrightarrow{\mathrm{r}}_{1}, \overrightarrow{\mathrm{r}}_{2}, t\right)}{\partial t} .
\end{aligned}
$$

The asymptotic solution of Eq. (6) $\left(\left|\vec{r}_{2}\right| \rightarrow \infty\right)$ is obtained from

$\left(\frac{p_{1}^{2}}{2 m}+V_{2}\left[\overrightarrow{\mathrm{r}}_{1}+\vec{\alpha}(t)\right]+\frac{1}{2 m}\left(\overrightarrow{\mathrm{p}}_{2}-e \overrightarrow{\mathrm{A}}\right)^{2}\right) \Phi=i \frac{\partial \Phi}{\partial t}$.

From now on we assume high-energy collision; in this case, neglecting the exchange between the electrons the solution for Eq. (7) can be written as the product of the exact solution for the $\overrightarrow{\mathrm{r}}_{2}$ variable and $\phi_{n}\left(\overrightarrow{\mathrm{r}}_{1}, t\right)$ :

$$
\begin{aligned}
\Phi \overrightarrow{\mathrm{k}}_{,}\left(\overrightarrow{\mathrm{r}}_{1}, \overrightarrow{\mathrm{r}}_{2}, t\right) \\
\quad=\exp \left\{i \overrightarrow{\mathrm{k}} \cdot\left[\overrightarrow{\mathrm{r}}_{2}-\vec{\alpha}(t)\right]\right\} \exp \left[-i\left(k^{2} / 2 m\right) t\right] \phi_{n}\left(\overrightarrow{\mathrm{r}}_{1}, t\right),
\end{aligned}
$$

where $\overrightarrow{\mathrm{k}}$ is the momentum of electron 2 , and $\phi_{n}\left(\overrightarrow{\mathrm{r}}_{1}, t\right)$ satisfies the equation

$$
\left(\frac{p_{1}^{2}}{2 m}+V_{2}\left[\overrightarrow{\mathrm{r}}_{1}+\vec{\alpha}(t)\right]\right) \phi_{n}\left(\overrightarrow{\mathrm{r}}_{1}, t\right)=i \frac{\partial \phi_{n}\left(\overrightarrow{\mathrm{r}}_{1}, t\right)}{\partial t} .
$$

No exact solution can be obtained for the states $\phi_{n}\left(\overrightarrow{\mathrm{r}}_{1}, t\right)$ and usually one assumes the so-called STA, which consists of neglecting completely $\vec{\alpha}(t)$ in that equation, and therefore $\phi_{n}\left(\overrightarrow{\mathrm{r}}_{1}, t\right)$ is taken as the solution of the field-free problem: $\phi_{n}^{(0)}\left(\vec{r}_{1}, t\right)$. That approximation is justifiable ${ }^{9}$ only for the region $r_{1} \gg \alpha_{0}=\chi / m \omega a$, where $a$ is the size of the bound system and $\chi=e a A_{0}$ is a dimensionless parameter whose square is a measure of the radiation intensity (values of $\chi$ up to $10^{-1}$ can be achieved presently in the laboratory). Since the bound functions $\phi_{n}$ are well concentrated within the region $r_{1} \sim a$, the STA is acceptable only if $a$ $\gg \alpha_{0}$.

An improvement over this approach consists of treating Eq. (9) within perturbation theory in the parameter $\alpha_{0}$. In the Appendix we present a discussion for a first-order perturbation theory, which is to be applied specifically to the hydrogen atom, and using this result we write the solution of Eq. (9) as

$$
\phi_{n}\left(\overrightarrow{\mathrm{r}}_{1}, t\right)=\phi_{n}^{(1)}\left(\overrightarrow{\mathrm{r}}_{1}\right) e^{-i \epsilon_{n} t} e^{-i \rho_{n} \cos \omega t},
$$

where $\phi_{n}^{(1)}$ is a linear combination of the unperturbed degenerate functions $\phi_{n}^{(0)}$ of energy $\epsilon_{n}$. To carry a simple notation we omit the superscript (1) in Eq. (10).

Using (10) in (8) the Green's 'unction is readily obtained:

$$
\begin{aligned}
& G\left(\overrightarrow{\mathrm{r}}_{1}^{\prime}, \overrightarrow{\mathrm{r}}_{2}^{\prime}, t^{\prime}, \overrightarrow{\mathrm{r}}_{1}, \overrightarrow{\mathrm{r}}_{2}, t\right)=\frac{i}{(2 \pi)^{3}} \sum_{n^{\prime}} \int d^{3} k \Phi_{\overrightarrow{\mathrm{k}}, n^{\prime}}^{*}\left(\overrightarrow{\mathrm{r}}_{1}^{\prime}, \overrightarrow{\mathrm{r}}_{2}^{\prime}, t^{\prime}\right) \Phi_{\overrightarrow{\mathrm{k}}_{, n^{\prime}}\left(\overrightarrow{\mathrm{r}}_{1}, \overrightarrow{\mathrm{r}}_{2}, t\right) \Theta\left(t-t^{\prime}\right)}=\frac{i}{(2 \pi)^{3}} \sum_{n^{\prime}} \int d^{3} k \phi_{n^{\prime}}^{*}\left(\overrightarrow{\mathrm{r}}_{1}^{\prime}\right) \phi_{n^{\prime}}\left(\overrightarrow{\mathrm{r}}_{1}\right) e^{i \overrightarrow{\mathrm{k}} \cdot\left(\overrightarrow{\mathrm{r}}_{2}-\overrightarrow{\mathrm{r}}_{2}^{\prime}\right)} \Theta\left(t-t^{\prime}\right) \\
& \times \exp \left[-i \int_{t^{\prime}}^{t} d t^{\prime \prime}\left(\epsilon_{n^{\prime}}+\frac{k^{2}}{2 m}-\frac{e}{m} \overrightarrow{\mathrm{k}} \cdot \overrightarrow{\mathrm{A}}\left(t^{\prime \prime}\right)-\rho_{n^{\prime}} \omega \sin \omega t^{\prime \prime}\right)\right] .
\end{aligned}
$$

The approximate solution of the Schrödinger equation associated with Eq. (6) is 


$$
\begin{aligned}
& \psi \overrightarrow{\mathrm{k}}_{0}, n\left(\overrightarrow{\mathrm{r}}_{1}, \overrightarrow{\mathrm{r}}_{2}, t\right)=\Phi_{\overrightarrow{\mathrm{k}}_{0}, n}\left(\overrightarrow{\mathrm{r}}_{1}, \overrightarrow{\mathrm{r}}_{2}, t\right)-\int d^{3} r_{1}^{\prime} d^{3} r_{2}^{\prime} d t^{\prime} G\left(\overrightarrow{\mathrm{r}}_{1}^{\prime}, \overrightarrow{\mathrm{r}}_{2}^{\prime}, t^{\prime}, \overrightarrow{\mathrm{r}}_{1}, \overrightarrow{\mathrm{r}}_{2}, t\right) \\
& \times\left[V_{1}\left(\overrightarrow{\mathrm{r}}_{2}^{\prime}\right)+V_{3}\left(\overrightarrow{\mathrm{r}}_{1}^{\prime}+\vec{\alpha}\left(t^{\prime}\right), \overrightarrow{\mathrm{r}}_{2}^{\prime}\right)\right] \psi_{\mathrm{k}_{0}, n}\left(\overrightarrow{\mathrm{r}}_{1}^{\prime}, \overrightarrow{\mathrm{r}}_{2}^{\prime}, t^{\prime}\right) \\
& =\Phi_{\overrightarrow{\mathrm{k}} 0, n}\left(\overrightarrow{\mathrm{r}}_{1}, \overrightarrow{\mathrm{r}}_{2}, t\right)-\frac{i}{(2 \pi)^{2}} \sum_{n^{\prime}} \int d^{3} k \cdot d^{3} \boldsymbol{r}_{1}^{\prime} d^{3} r_{2}^{\prime} d t^{\prime} \phi_{n^{\prime}}^{*}\left(\overrightarrow{\mathrm{r}}_{1}^{\prime}\right) \phi_{n^{\prime}}\left(\overrightarrow{\mathrm{r}}_{1}\right) e^{i \overrightarrow{\mathrm{k}} \cdot\left(\overrightarrow{\mathrm{r}}_{2}-\overrightarrow{\mathrm{r}}_{2}^{\prime}\right)} \\
& \times \exp \left\{-i\left[\eta\left(\overrightarrow{\mathrm{k}}, n^{\prime}, t\right)-\eta\left(\overrightarrow{\mathrm{k}}, n^{\prime}, t^{\prime}\right)\right]\right\}\left\lfloor V_{1}+V_{3}\right] \psi_{\mathrm{k}_{0}, n}\left(\overrightarrow{\mathrm{r}}_{1}^{\prime}, \overrightarrow{\mathrm{r}}_{2}^{\prime}, t^{\prime}\right),
\end{aligned}
$$

where we have defined

$\eta\left(\overrightarrow{\mathrm{k}}, n^{\prime}, t\right) \equiv\left(\frac{k^{2}}{2 m}+\epsilon_{n^{\prime}}\right) t+e \frac{\overrightarrow{\mathrm{A}}_{0} \cdot \overrightarrow{\mathrm{k}}}{m \omega} \sin \omega t+\rho_{n^{\prime}} \cos \omega t$

or, in a more convenient form,

$$
\begin{aligned}
\eta\left(\overrightarrow{\mathrm{k}}, n^{\prime}, t\right) \equiv\left(k^{2} / 2 m-k_{0}^{2} / 2 m+\epsilon_{n}-\epsilon_{n^{\prime}}\right) t \\
+\beta\left(\overrightarrow{\mathrm{k}}_{0}, \overrightarrow{\mathrm{k}}, n, n^{\prime}, t\right)+\rho_{n^{\prime}} \cos \omega t,
\end{aligned}
$$

with

$$
\beta\left(\overrightarrow{\mathrm{k}}_{0}, \overrightarrow{\mathrm{k}}, n, n^{\prime}, t\right) \equiv\left(\frac{k_{0}^{2}}{2 m}+\epsilon_{n}\right) t+\vec{\alpha}_{0} \cdot \overrightarrow{\mathrm{k}} \sin \omega t .
$$

Using the periodicity of the function ${ }^{2,3}$

$$
\begin{aligned}
\psi_{\overrightarrow{\mathrm{k}}, \overrightarrow{\mathrm{k}}_{0}}^{n^{\prime}, n}\left(\overrightarrow{\mathrm{r}}_{1}, \overrightarrow{\mathrm{r}}_{2}, t\right) & \equiv \exp \left[i \beta\left(\overrightarrow{\mathrm{k}}_{0}, \overrightarrow{\mathrm{k}}, n, n^{\prime}, t\right)\right] \psi_{\overrightarrow{\mathrm{k}}_{0}, n}\left(\overrightarrow{\mathrm{r}}_{1}, \overrightarrow{\mathrm{r}}_{2}, t\right) \\
& =\sum_{j=-\infty}^{\infty} e^{i j \omega t} \psi_{k_{0}{ }^{j}, n^{\prime} n}^{j}\left(\overrightarrow{\mathrm{r}}_{1}, \overrightarrow{\mathrm{r}}_{2}\right)
\end{aligned}
$$

and the relations

$$
\begin{aligned}
& e^{i \alpha \sin \omega t}=\sum_{l=-\infty}^{\infty} J_{l}(\alpha) e^{i l \omega t} e^{i \alpha \cos \omega t} \\
& =\sum_{l=-\infty}^{\infty}(i)^{l} J_{l}(\alpha) e^{i l \omega t}, \\
& V_{3}\left(\overrightarrow{\mathrm{r}}_{1}+\vec{\alpha}, \overrightarrow{\mathrm{r}}_{2}\right)=e^{+\vec{\alpha} \cdot \vec{\nabla}_{1}} V_{3}\left(\overrightarrow{\mathrm{r}}_{1}, \overrightarrow{\mathrm{r}}_{2}\right) \\
& =\sum_{t=-\infty}^{\infty}\left[J_{l}\left(\vec{\alpha}_{0} \cdot \overrightarrow{\mathrm{p}}_{1}\right) V_{3}\left(\overrightarrow{\mathrm{r}}_{1}, \overrightarrow{\mathrm{r}}_{2}\right)\right] e^{i \omega t t}
\end{aligned}
$$

the integration in $t^{\prime}$ of Eq. (13) can be readily performed, and we get

$$
\begin{aligned}
\psi \overrightarrow{\mathrm{k}}_{0, n}=\Phi_{k_{0}, n}+\frac{2 m}{(2 \pi)^{3}} \sum_{\substack{n^{\prime} l \\
j s}}(i)^{l} \int & d^{3} k d^{3} r_{1}^{\prime} d^{3} r_{2}^{\prime} \phi_{n^{\prime}}^{*}\left(\overrightarrow{\mathrm{r}}_{1}^{\prime}\right) \phi_{n^{\prime}}\left(\overrightarrow{\mathrm{r}}_{1}\right) e^{i \overrightarrow{\mathrm{k}} \cdot\left(\overrightarrow{\mathrm{r}}_{2}-\overrightarrow{\mathrm{r}}_{2}^{\prime}\right)} e^{-i \eta\left(\overrightarrow{\mathrm{k}}, n^{\prime}, t\right)} J_{l}\left(\rho_{n^{\prime}}\right) \\
& \times\left[J_{s}\left(\vec{\alpha}_{0} \cdot \overrightarrow{\mathrm{p}}_{1}\right) V_{3}\left(\overrightarrow{\mathrm{r}}_{1}^{\prime}, \overrightarrow{\mathrm{r}}_{2}^{\prime}\right)\right] \psi_{\overrightarrow{\mathrm{k}}_{0}, \overrightarrow{\mathrm{k}}_{,} n^{\prime}, n}\left(\overrightarrow{\mathrm{r}}_{1}^{\prime}, \overrightarrow{\mathrm{r}}_{2}^{\prime}\right) \frac{1}{k^{2}-k^{2}(\nu)},
\end{aligned}
$$

where we do not include the term $V_{1}$ since it does not contribute for the inelastic transition amplitude. Energy conservation comes from the integration over $t^{\prime}$ [the $\delta$ function in (20)], since

$$
\frac{k^{2}(\nu)}{2 m}=\frac{k_{0}^{2}}{2 m}-\epsilon_{n^{\prime}}+\epsilon_{n}-\nu \omega \text { with } \nu=j-l+s .
$$

The parameter $\nu<0$ represents absorption, and $\nu>0$ represents emission of $|\nu|$ photons by the $e-\mathrm{H}$ system during the scattering process.

After performing the integration in $\vec{k}$ we obtain

$\lim _{|\overrightarrow{\mathrm{r}}| \rightarrow \infty} \psi_{\overrightarrow{\mathrm{k}}_{0}, n}\left(\overrightarrow{\mathrm{r}}_{1}, \overrightarrow{\mathrm{r}}_{2}, t\right)=\Phi_{\overrightarrow{\mathrm{k}}_{0}, n^{n}}\left(\overrightarrow{\mathrm{r}}_{1}, \overrightarrow{\mathrm{r}}_{2}, t\right)+\sum_{n^{\prime} \nu} f_{n, n^{\prime}}^{\overrightarrow{\mathrm{k}}_{0}, \overrightarrow{\mathrm{k}}(\nu)}(\theta, \phi) e^{+i \overrightarrow{\mathrm{k}}(\nu) \cdot \vec{\alpha}(t)} \exp \left(-i \frac{k^{2}(\nu)}{2 m} t\right) \phi_{n^{\prime}}\left(\overrightarrow{\mathrm{r}}_{1}, t\right) \frac{e^{i k(\nu) r_{2}}}{r^{2}}$,

where

$$
\begin{aligned}
f_{n, n^{\prime}}^{\vec{k}_{0}, \vec{k}(\nu)}(\Omega)=-\frac{m}{2 \pi} \sum_{l, s=-\infty}^{\infty}(-1)^{s}(i)^{l} \int & d^{3} r_{1}^{\prime} d^{3} r_{2}^{\prime} e^{-i \overrightarrow{\mathrm{k}}(\nu) \cdot \overrightarrow{\mathrm{r}}_{2}^{\prime}} \phi_{n^{\prime}}^{*}\left(\overrightarrow{\mathrm{r}}_{1}^{\prime}\right) J_{l}\left(\rho_{n^{\prime}}\right) \\
& \times\left[J_{s}\left(\vec{\alpha}_{0} \cdot \overrightarrow{\mathrm{p}}_{1}\right) V_{3}\left(\overrightarrow{\mathrm{r}}_{1}^{\prime}, \overrightarrow{\mathrm{r}}_{2}^{\prime}\right)\right] \Psi_{\overrightarrow{\mathrm{k}}_{0}, \overrightarrow{\mathrm{k}}(\nu), n^{\prime}, n}^{\prime+l-s}\left(r_{1}^{\prime}, r_{2}^{\prime}\right),
\end{aligned}
$$

and

$$
\overrightarrow{\mathrm{k}}(\nu)=k(\nu) \hat{r}_{2} .
$$


From (22), $f_{n, n^{\prime}}^{\overrightarrow{\mathrm{k}}_{0}, \overrightarrow{\mathrm{k}}(\nu)}$ is identified immediately with the scattering amplitude of the process in which the scattered electron goes from state $\overrightarrow{\mathrm{k}}_{0}$ into state $\overrightarrow{\mathrm{k}}(\nu)$. The atom suffers a transition from state $n$ into state $n^{\prime}$, with simultaneous absorption $(\nu<0)$ or emission $(\nu>0)$ of $|\nu|$ photons by the system.

The scattering amplitude (23) can be written in a more convenient expression if we use Eq. (17) to get

$$
\psi_{k_{0} k(\nu), n n^{\prime}}^{\nu+l-s}\left(\overrightarrow{\mathrm{r}}_{1}, \overrightarrow{\mathrm{r}}_{2}\right)=-\frac{\omega}{2 \pi} \int_{0}^{2 \pi / \omega} d t e^{-i \omega t(\nu+l-s)} \psi_{\overrightarrow{\mathrm{k}}(\nu), \overrightarrow{\mathrm{k}}}^{n^{\prime}, n}\left(\overrightarrow{\mathrm{r}}_{1}, \overrightarrow{\mathrm{r}}_{2}, t\right)
$$

which leads to

$$
f_{n, n^{\prime}}^{k_{0}, k(\nu)}(\Omega)=-\frac{m \omega}{(2 \pi)^{2}} \sum_{l s p}(-1)^{p}(i)^{l+p} \int_{0}^{2 \pi / \omega} d t e^{i(p-l+s) \omega t} J_{l}\left(\rho_{n^{\prime}}\right) J_{p}\left(\rho_{n^{\prime}}\right)\left\langle\Phi_{\overrightarrow{\mathrm{k}}(\nu), n^{\prime}}\left|J_{s}\left(\vec{\alpha}_{0} \cdot \overrightarrow{\mathrm{p}}_{1}\right) V_{3}\left(\overrightarrow{\mathrm{r}}_{1}, \overrightarrow{\mathrm{r}}_{2}\right)\right| \psi_{\overrightarrow{\mathrm{k}}_{0}, n}\right\rangle
$$

Using (18) and (19), summations over $s p l$ can be performed immediately, resulting in

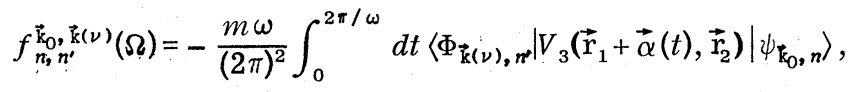

where the symbol $\langle||\rangle$ denotes integration over $\overrightarrow{\mathrm{r}}_{1}$ and $\overrightarrow{\mathrm{r}}_{2}$.

The usual STA is obtained assuming $\rho_{n} \rightarrow 0$ and $\phi_{n}^{(1)} \rightarrow \phi_{n}^{(0)}$ in the asymptotic solution of Eq. (7):

$$
\Phi_{\overrightarrow{\mathrm{k}}, n}\left(\overrightarrow{\mathrm{r}}_{1}, \overrightarrow{\mathrm{r}}_{2}, t\right)=e^{i \overrightarrow{\mathrm{k}} \cdot[\overrightarrow{\mathrm{r}}-\vec{\alpha}(t)]} e^{-\left(k^{2} / 2 m\right) t} \phi_{n}^{(0)}\left(\overrightarrow{\mathrm{r}}_{1}\right) e^{-i \epsilon_{n} t}
$$

and the scattering amplitude in the STA is given by Eq. (27), where, for consistency, $V_{3}\left(\vec{r}_{1}+\vec{\alpha}(t)\right.$, $\overrightarrow{\mathrm{r}}_{2}$ ) should be approximated by $V_{3}\left(\overrightarrow{\mathrm{r}}_{1}, \overrightarrow{\mathrm{r}}_{2}\right)$, with the above expression for the $\Phi_{\overrightarrow{\mathrm{k}}(\nu), n^{\prime}}$.

\section{MOMENTUM TRANSLATION AND THE KELDYSH APPROXIMATIONS}

The same procedure we have described in Sec. II can be used to obtain an expression for the scattering amplitude when one uses the MTA or the $\mathrm{KA}$ to describe the bound states of the hydrogen atom. For the $\mathrm{MTA}^{13}$ we have the asymptotic $\left(\left|\mathrm{r}_{2}\right|-\infty\right)$ solution for Hamiltonian (1):

$$
\begin{aligned}
\Phi_{\overrightarrow{\mathrm{k}}, n}= & e^{i \overrightarrow{\mathrm{k}} \cdot\left[\overrightarrow{\mathrm{r}}_{2}-\vec{\alpha}(t)\right]} e^{-i\left(k^{2} / 2 m\right) t} e^{i \vec{e} \overrightarrow{\mathrm{A}}(t) \cdot \overrightarrow{\mathrm{r}}} \\
& \times \phi_{n}^{(0)}(\overrightarrow{\mathrm{r}}) e^{-i \epsilon_{n} t}
\end{aligned}
$$

and for the Keldysh approximation ${ }^{3,14}$

$$
\begin{aligned}
\phi_{\overrightarrow{\mathrm{k}}, n}= & e^{i \overrightarrow{\mathrm{k}} \cdot\left[\overrightarrow{\mathrm{r}}_{2}-\vec{\alpha}(t)\right]} e^{-i\left(k^{2} / 2 m\right) t} e^{-i \sigma_{n} \cos \omega t} \\
& \times \phi_{n}^{(0)}(\overrightarrow{\mathrm{r}}) e^{i \epsilon_{n} t},
\end{aligned}
$$

where $\sigma_{n}$ is the Stark-effect parameter defined by Keldysh. ${ }^{14}$

No unitary transformation is applied to the Hamiltonian in Eq. (1) so that for these cases the scattering amplitude takes the for $\mathrm{m}^{3}$

$$
\begin{aligned}
& f_{n, n^{\prime}}^{\vec{k}_{0}, \vec{k}(\nu)}(\Omega) \\
& =-\frac{m \omega}{(2 \pi)^{2}} \int_{0}^{2 \pi / \omega} d t\left\langle\Phi_{\overrightarrow{\mathrm{k}}(\nu), n^{\prime}}\left|V_{3}\left(\overrightarrow{\mathrm{r}}_{1}, \overrightarrow{\mathrm{r}}_{2}\right)\right| \psi_{\overrightarrow{\mathrm{k}}_{0}, n}\right\rangle .
\end{aligned}
$$

Equation (30) clearly shows that the MTA is not adequate to treat this type of problem since the corresponding scattering amplitude is completely independent of the effect of the EMF on the bound states. The whole contribution to multiphoton processes appears from the interaction between the EMF and electron 2. The MTA has been under criticism, ${ }^{15,16}$ and a word must be said about the objections concerning this approximation. CohenTannoudji et al. ${ }^{15}$ and Decoster ${ }^{16}$ argue that the multiphoton amplitudes given by this theory cannot be considered as reliable. The condition of validity of the MTA according to Decoster ${ }^{16}$ is basically $\nu \omega \ll \epsilon$ (instead of $\omega \ll \epsilon$ ) for all field intensities. This is the case here, if most of the energy involved in the atomic transition is provided by the colliding electron and not by the EMF. Our results show that even for this case the MTA does not introduce any modification in the bound state. We should note that in the present treatment the asymptotic region is characterized by no interaction between the electron and the atom, but both always interact with the EMF, contrary to Reiss' ${ }^{13}$ treatment, where no collision process is involved, and the asymptotic condition is given by an adiabatic turning on and off of the EMF for $t=-\infty$ and $t=+\infty$.

\section{RESULTS IN THE BORN APPROXIMATION}

To better compare the different approximations previously discussed we will assume high-energy collisions and treat the scattering amplitude in the Born approximation, substituting the exact solution $\psi \overrightarrow{\mathrm{k}}_{0}, n$ by its asymptotic expression $\Phi_{\mathrm{k}_{0}, n^{\prime}}$ within the specific approximations. Firstly, we present the calculations for the scattering amplitude within the STA1 and from it trivially obtain 
the other transition amplitudes.

For convenience we will introduce the following quantities:

$$
\begin{aligned}
& V_{3}(\overrightarrow{\mathrm{r}})=\frac{1}{(2 \pi)^{3}} \int \tilde{V}_{3}(\overrightarrow{\mathrm{K}}) e^{-i \overrightarrow{\mathrm{K}} \cdot \overrightarrow{\mathrm{r}}} d^{3} K, \\
& F_{n^{\prime} n}^{(i)}(K)=\int e^{-i \overrightarrow{\mathrm{K}} \cdot \overrightarrow{\mathrm{r}}} \phi_{n^{\prime}}^{(i) *(\overrightarrow{\mathrm{r}}) \phi_{n}^{(i)}(\overrightarrow{\mathrm{r}}),}
\end{aligned}
$$

where $(i)$ denotes the order of approximation for the bound states in the EMF, and

$$
\overrightarrow{\mathrm{q}}(\vec{\nu})=\overrightarrow{\mathrm{k}}(\nu)-\overrightarrow{\mathrm{k}}_{0},
$$

the transferred momentum.

\section{A. First-order space-translation approximation (STA1)}

Using the above definitions together with Eq. (27) and (19), we get

$$
\begin{aligned}
{\left[f_{n^{\prime} n}^{\overrightarrow{\mathrm{k}}_{0}, \overrightarrow{\mathrm{k}}(\nu)}(\Omega)\right]_{\mathrm{STA} 1}^{\mathrm{BA}}=} & -\frac{m \omega}{(2 \pi)^{2}} \tilde{V}_{3}(\overrightarrow{\mathrm{q}}(\nu)) F_{n^{\prime} n}^{(1)} \\
& \times \sum_{s=-\infty}^{\infty} J_{s}\left[\vec{\alpha}_{0} \cdot \overrightarrow{\mathrm{q}}(\nu)\right] \int_{0}^{2 \pi} d t \exp \left[i\left(\frac{k^{2}(\nu)}{2 m}+\epsilon_{n^{\prime}}-\frac{k_{0}^{2}}{2 m}+\epsilon_{n}+s \hat{\omega}\right) t\right] \exp \left[-i \overrightarrow{\alpha_{0}} \cdot \overrightarrow{\mathrm{q}}(\nu) \sin \omega t\right] \\
& \times \exp \left[i\left(\rho_{n^{\prime}}-\rho_{n}\right) \cos \omega t\right] .
\end{aligned}
$$

We define

$$
\vec{\alpha}_{0} \cdot \vec{q}(\nu) \equiv \gamma(\nu)
$$

and

$$
f_{0}^{(i)}(\overrightarrow{\mathrm{q}}(\nu)) \equiv-(m / 2 \pi) \tilde{V}_{3}(\overrightarrow{\mathrm{q}}(\nu)) F_{n^{\prime} n}^{(i)}(\overrightarrow{\mathrm{q}}(\nu)) \delta\left[k^{2}(\nu) / 2 m-k_{0}^{2} / 2 m+\epsilon_{n^{\prime}}-\epsilon_{n}+\epsilon_{n}+\nu \omega\right] .
$$

Integration over $t$ is readily done using Eq. (18) and we obtain

$$
\left[f_{n^{\prime} n}^{\vec{k}_{0}, \overrightarrow{\mathrm{k}}(\nu)}(\Omega)\right]_{\mathrm{STA}^{\mathrm{B} A} 1}^{\mathrm{BA}}=f_{0}^{(1)}(\overrightarrow{\mathrm{q}}(\nu)) \sum_{s=-\infty}^{\infty} \sum_{l=m}^{\infty}(i)^{l} J_{s}(-\gamma(\nu)) J_{\nu-s-l}(\gamma(\nu)) J_{l}\left(\rho_{n},-\rho_{n}\right) \text {. }
$$

Finally, using Eq. 8.530 of Ref. 17 the scattering amplitude (35) is reduced to a simple form (except for an irrelevant phase):

$$
\left[f_{n_{n}^{\prime}}^{\overrightarrow{\mathrm{k}}_{n}, \overrightarrow{\mathrm{k}}(\nu)}(\Omega)\right]_{\mathrm{STA} 1}^{\mathrm{BA}}=f_{0}^{(1)}(q(\nu)) J_{\nu}\left(\rho_{n},-\rho_{n}\right) .
$$

B. Zero-order space translation approximation (STA)

As previously remarked the STA is obtained from the STA1 by taking $\rho_{n}=\rho_{n},=0$ and $\vec{\alpha}(t)=0$ in $\mathrm{Eq}$. (27). In this case Eq. (36) reduces to

$$
\left[f_{n^{\prime} n}^{\overrightarrow{\mathrm{k}}_{\mathrm{o}} \overrightarrow{\mathrm{k}}(\nu)}(\Omega)\right]_{\mathrm{STA}}^{\mathrm{BA}}=f_{0}^{(0)}(\overrightarrow{\mathrm{q}}(\nu)) J_{\nu}(\gamma(\nu)) \text {. }
$$

\section{Keldysh approximation}

A comparison between (27) and (30) shows that for the KA as well as for the MTA no sum over $s$ as in Eq. (35) is present in the scattering amplitude. To obtain an expression for the scattering amplitude in the KA from (35), we simply ignore the sum in $s$ and substitute $\rho_{n}$ by $\sigma_{n}$ as given by (29):

$$
\begin{aligned}
& {\left[f_{n^{\prime} n}^{\overrightarrow{\mathrm{k}}_{0}, \overrightarrow{\mathrm{k}}(\nu)}(\Omega)\right]_{\mathrm{KA}}^{\mathrm{BA}}} \\
& \quad=f_{0}^{(0)}(\overrightarrow{\mathrm{q}}(\nu)) J_{\nu}\left\{\left[(\gamma(\nu))^{2}+\left(\sigma_{n^{\prime}}-\sigma_{n}\right)^{2}\right]^{1 / 2}\right\} .
\end{aligned}
$$

\section{Momentum-translation approximation}

From Eqs. (28) and (30), the MTA can be readily derived from (35), since no summation over $s$ or $l$ must be performed, resulting in

$$
\left[f_{n^{\prime} n}^{\overrightarrow{\mathrm{k}}_{0}, \overrightarrow{\mathrm{k}}(\nu)}(\Omega)\right]_{\mathrm{MTA}}^{\mathrm{BA}}=f_{0}^{(0)}(\overrightarrow{\mathrm{q}}(\nu)) J_{\nu}(\gamma(\nu)) .
$$

It is interesting to observe that the final expressions for the scattering amplitude in all the approximations considered in the present work were brought into the product of a factor which reauces to the field-free scattering amplitude for $\nu=0$ and a Bessel function of order $\nu$, whose argument depends on the specific approximation. As we mentioned previously, the KA was also used by Bhakar and Choudhury for the treatment of this problem, and their result in the BA differs from (38) only by the argument of the Bessel function; this is due to the fact that they have mistakenly chosen the same phase for the vector potential and the electric field. Our result (38) is in complete agreement with Perelman and Kovarskii's [Eq. (6) of Ref. 1].

For small-field intensities, all the approximations we have considered give a dependence for the differential cross section with the number of photons involved in the process of the form 
$d \sigma / d \Omega \propto \chi^{2|\nu|}$, where $\chi^{2}$ is the intensity parameter of the EMF, which clearly shows that for weak fields multiphoton processes are not relevant, as it is well known.

\section{DISCUSSIONS AND CONCLUSIONS}

It is clear that our results can be separated into two physically different treatments: the MTA and STA are not sensitive to the the structural modifications of the bound states caused by the EMF, and therefore can not be considered as adequate to treat the intense EMF limit as the STA1 and KA. As discussed in the Appendix, both the STA1 and $\mathrm{KA}$ are valid only within the dipole approximation, i.e., $\lambda \gg a$. This is the only restriction for the $\mathrm{KA}$, while for the STA1 besides that condition we must have $a \gg \alpha_{0}=e E_{0} / m \omega^{2}$, therefore the region of validity of the $\mathrm{KA}$ is less restricted than that of the STA1. The only manner to determine the best one is a comparison with experimental results for an EMF within their common region of validity.

For the hydrogen atom, $a \sim 10^{-8} \mathrm{~cm}$, and typical values of $\alpha_{0}$ are $10^{-7} \mathrm{~cm}$ for a $\mathrm{CO}_{2}$ laser $\left(\lambda_{\mathrm{CO}_{2}}\right.$ $\left.=10^{-5} \mathrm{~cm}\right)$ and $10^{-9} \mathrm{~cm}$ for a Nd-glass laser $\left(\lambda_{\mathrm{Nd}}\right.$ $\left.=1.06 \times 10^{-6} \mathrm{~cm}\right)$. Both can be treated within the dipole approximation, however, as discussed, the STA1 is not adequate for the $\mathrm{CO}_{2}$ laser.

In the treatment of vibronic transitions in molecules, the large mass of the system yields values for $\alpha_{0}$ orders of magnitude smaller than those presented here for electronic transitions, and a STA-type treatment may become valid even for high-intensity or low-frequency laser fields.

\section{ACKNOWLEDGMENTS}

We acknowledge the financial support of the Brazilian Agencies Conselho Nacional de Desenvolvimento Cientifico e Tecnológico and Financiadora de Estudos e Projetos.

\section{APPENDIX: HYDROGEN-ATOM BOUND STATES IN AN ELECTROMAGNETIC FIELD}

Under a space-translation transformation (4), the Schrödinger equation for the hydrogen atom in 'an $\mathrm{EMF}$ is' written

$$
\left(\frac{p^{2}}{2 m}+V[\overrightarrow{\mathrm{r}}+\vec{\alpha}(t)]\right) \phi(\overrightarrow{\mathrm{r}}, t)=i \frac{\partial-\phi(\overrightarrow{\mathrm{r}}, t)}{\partial t} .
$$

In the usual STA $V[\overrightarrow{\mathrm{r}}+\vec{\alpha}(t)] \sim V(\overrightarrow{\mathrm{r}})$. Let us consider the expansion

$$
V(\overrightarrow{\mathrm{r}}+\vec{\alpha}) \simeq V(\overrightarrow{\mathrm{r}})+(\vec{\alpha} \cdot \vec{\nabla}) V(\overrightarrow{\mathrm{r}})+\cdots
$$

and treat the second term in (A2) as a perturbation to the field-free hydrogen-atom Hamiltonian. We call this approximation the first-order STA

(STA1).

In this case (A1) is approximated by

$$
\left(\frac{p^{2}}{2 m}-\frac{e^{2}}{r}-\frac{e^{3}}{m \omega^{2}} \frac{\overrightarrow{\mathrm{E}} \cdot \overrightarrow{\mathrm{r}}}{r^{3}}\right) \phi^{(1)}(\overrightarrow{\mathrm{r}}, t)=i \frac{\partial \phi^{(1)}(\overrightarrow{\mathrm{r}}, t)}{\partial t},
$$

where

$$
E_{1}(t)=-\frac{\partial \vec{A}}{\partial t}=\omega \vec{A}_{0} \sin \omega t \equiv \overrightarrow{\mathrm{E}}_{0} \sin \omega t
$$

The wave function of the $n$th level ( $g$-fold degenerate) of the hydrogen atom, corrected to first order due to the EMF can be written

$$
\phi_{n}^{(1)}(\vec{r}, t)=\sum_{\alpha=1}^{g} a_{n \alpha}(t) \phi_{n \alpha}^{(0)}(\vec{r}) e^{-i \epsilon_{n} t},
$$

where one easily obtains

$$
a_{n \alpha}(t)=C_{n \alpha} \exp \left(-i \rho_{n} \cos \omega t\right)
$$

and $\rho_{n}$ is one of the roots of the secular equation

$$
\begin{aligned}
& \operatorname{det}\left|\omega \rho_{n} \delta_{\alpha \beta}+V_{\alpha \beta}\right|=0, \\
& V_{\alpha \beta}=\left(e^{3} / m \omega^{2}\right)\left\langle\alpha\left|\overrightarrow{\mathrm{E}}_{0} \cdot \overrightarrow{\mathrm{r}} / \overrightarrow{\mathrm{r}}^{3}\right| \beta\right\rangle,
\end{aligned}
$$

and

$$
\phi_{n}^{(1)}(\overrightarrow{\mathrm{r}}, t)=\sum_{\alpha=1}^{g} C_{n \alpha} \phi_{n \alpha}^{(0)}(\overrightarrow{\mathrm{r}}) e^{-i \epsilon_{n} t} \exp \left(-i \rho_{n} \cos \omega t\right) .
$$

This result is very similar to the one obtained by Kovarskii and Perelman ${ }^{18}$ where

$$
V_{\alpha \beta}=-e\left\langle\alpha\left|\overrightarrow{\mathrm{E}}_{0} \cdot \overrightarrow{\mathrm{r}}\right| \beta\right\rangle
$$

is obtained directly from the dipole approximation for the interaction Hamiltonian.

The Keldysh approximation does not include the sum over the degenerate levels for the spatial variables, but has the same time-dependence structure.
* Permanent address: Centro de Investigacion y de Estudios Avanzados de IPN, Mexico 14, DF, Mexico. ${ }^{1}$ N. F. Perelman and V. A. Kovarskii, Sov. Phys. JETP 36, 436 (1973).

${ }^{2}$ N. M. Kroll and K. M. Watson, Phys. Rev. A $\underline{8}, 804$ (1973).

${ }^{3}$ B. S. Bhakar and B. J. Choudhury, J. Phys. B 7, 1866 (1974).
${ }^{4}$ N. D. Blashkar, B. Jaduszliwer, and B. Bederson, Phys. Rev. Lett. 38, 14 (1977).

${ }^{5}$ T. V. Hertel and W. Stoll, J. Phys. B 7 , 570 (1974); J. Appl. Phys. 47, 214 (1976).

${ }^{6} \mathrm{~J}$. Macek and I. V. Hertel, J. Phys. B 7, 2173 (1974).

'J. J. Gersten and M. H. Mittleman, Phys. Rev. A 13, 123 (1976).

${ }^{8}$ M. H. Mittleman, Phys. Rev. A 14, 1338 (1976); 15, 
1335(E) (1977).

${ }_{9}^{9}$ F. H. M. Faisal, J. Phys. B 6 , L89 (1973); 6, L312 (1973).

${ }^{10}$ M. Mohan, Phys. Lett. 50A, 283 (1974).

${ }_{11}^{11}$ M. Mohan, International Center for Theoretical Physics-Trieste Internal Report No. IC/77/23 (unpublitshed).

${ }^{12}$ W. C. Henneberger, Phys. Rev. Lett. 21, 838 (1968).

${ }^{13}$ H. R. Reiss, Phys. Rev. A $1,803(1970)$.
${ }^{14}$ L. V. Keldysh, Sov. Phys. JETP 20, 1307 (1965).

${ }^{15} \mathrm{C}$. Cohen-'Tannoudji, J. Dupont-Roc, C. Fabre, and G. Grynbey, Phys. Rev. A 8 , 2747 (1973).

${ }^{16}$ A. Decoster, Phys. Rev. A 9, 1446 (1974).

${ }^{17}$ I. S. Gradshteyn and I. M. Ryzhik, Table of Integrals, Series and Products (Academic, New York, 1965), p. 979.

${ }^{18}$ V. A. Kovarskii and N. F. Perelman, Sov. Phys. JETP 34,738 (1972). 